\title{
Perspectives on Strategies to Direct Elastic Matrix Assembly
}

\section{Chris A. Bashur and Anand Ramamurthi*}

Department of Biomedical Engineering, Cleveland Clinic, Cleveland, Ohio, USA

\begin{abstract}
Progress in tissue engineering clinically useful replacements for soft, elastic tissues is presently constrained by poor elastogenicity of most adult cell types, and difficulties in replicating the biocomplexity of elastic matrix assembly that occurs primarily in the fetal and neonatal stages. With recent progress in being able to enhance elastin precursor (tropoelastin) synthesis by adult cell types, the present emphasis in the field has shifted to developing strategies to address the other, equally important, if not more critical issues such as extremely poor recruitment and crosslinking of tropoelastin, and the need to direct the organization of crosslinked elastin deposits into matrix structures (e.g., aligned, spatially-oriented fibers) so as to be able to replicate the mechanical anisotropy of native tissues. This editorial provides insight into potential strategies to address these challenges and the key factors that are likely to influence their outcomes.
\end{abstract}

\section{Introduction}

Emerging tissue engineering technologies proffer significant potential to fabricate tissue and organ replacements, seemingly on demand, using autologous, patient-derived cells. However, replicating the complex matrix composition and architecture of various soft tissues, specifically those that typically contain permenant/stable cell types (e.g., cardiac and vascular cells), is a major challenge in the field. Specifically, one yet unsurmounted challenge is the inability to coax such adult cell types to synthesize sufficient elastin and biomimetically assemble these precursors into mature elastic fibers and matrix structures. Elastic fibers, which are composed of microfibrillar components that form pre-scaffolds onto which elastin protein deposits and is crosslinked, critically maintain native tissue structure, allow them to recoil following stretch, and enable biomechanical transduction of contacting cells [1-4]. Thus, the incorporation of such fibers into engineered tissue constructs and the ability to organize these fibers into higher-order structures (e.g., sheets, lamellae) are critical to ensuring proper biological and mechanical tissue function.

Developing technologies to mimic physiologic elastic matrix assembly is an area of active, ongoing research that has been comprehensively discussed in several recent reviews [5,6]. This assembly process involves several steps of coordinated intracellular and extracellular activities, typically directed by smooth muscle cells or fibroblasts, which include (a) synthesis of elastin precursor molecules (i.e., tropoelastin), (b) their transport to the extracellular space, (c) binding of the cell-surface elastin-laminin receptor (ELR), (d) coordinated engagement of a galactolectin binding site present on the elastin binding protein (EBP) of the ELR by glycoprotein (e.g., fibrillin) microfibrils, release of tropoelastin onto these pre-scaffolds for crosslinking by lysyl oxidase (LOX) enzymes into matrix deposits, and (e) radial and longitudinal growth of these deposits to form mature fibers.

Research to date suggests that the greatest impediments to engineering elastic matrix-rich tissues in vitro are the poor tropoelastin mRNA expression and translation by most cell types beyond the neonatal stage, $[7,8]$ barring certain exceptions such as smooth muscle cells (SMCs) of the bladder [9] and female reproductive tissues, [10] and their inefficiency in crosslinking tropoelastin and organizing the matrix deposits into fibers and fibrous elastic matrix superstructures [11]. In recent years, there has been significant progress in the ability to enhance elastin precursor synthesis multi-fold by stimulating cells with biological cell signaling factors, choosing appropriate scaffold composition, and incorporating bioreactors. Promising biological factors include cyclic GMP, [12] fetal calf serum, [13] insulin-like growth factor 1 (IGF-1), [14] transforming growth factor $\beta 1$ (TGF- $\beta$ ), [15] aldosterone, [16] retinoic acid, [17] and hylauronan oligomers (HA-o) [18]. Scaffold engineering strategies include controlling chemistry (e.g. the incorporation of fibrin into collagen-containing scaffolds that impede elastin biosynthesis), [19] adding factors (e.g. aldosterone) to restrict typically prolific cellular collagen synthesis that follows cell seeding on most synthetic scaffolding types, [19-21] tuning scaffold compliance (i.e, rendering the substrate not too stiff nor not too compliant improves cellular elastin biosynthesis), [22,23] and providing a three-dimensional scaffold (i.e., compared to cells cultured on smooth 2-D films, cells within porous 3-D scaffolds show improved cell adherence and tropoelastin synthesis, and reduced collagen synthesis) [24]. In addition, biomechanical transduction of cells via application of cyclic mechanical stretch has been shown to improve matrix deposition [23]. While promising, the collective body of work in this field has indicated that the total amount of elastic matrix that can be generated in culture is highly insufficient to create tissue replacements of finite sizes, fit for clinical use. To overcome this challenge, it is necessary to more efficiently recruit and process (i.e., coacervate, crosslink, and engage with glycoprotein microfibrils) tropoelastin precursors to significantly enhance the fractional yield of elastic matrix and stabilize the elastic matrix structures against rapid biodegradation. Further, spatial orientation of and proper alignment among elastic fibers must be achieved in order for the engineered tissue constructs to replicate the anisotropic mechanics of the elastic tissue types they are intended to replace. Strategies to enhance these

*Corresponding author: Anand Ramamurthi, Department of Biomedica Engineering, Cleveland Clinic, Cleveland, Ohio, USA, E-mail: ramamua@ccf.org

Received October 09, 2011; Accepted November 15, 2011; Published November 17, 2011

Citation: Bashur CA, Ramamurthi A (2011) Perspectives on Strategies to Direct Elastic Matrix Assembly. J Tissue Sci Eng 2:106e. doi:10.4172/21577552.1000106e

Copyright: (c) 2011 Bashur CA, et al. This is an open-access article distributed under the terms of the Creative Commons Attribution License, which permits unrestricted use, distribution, and reproduction in any medium, provided the original author and source are credited. 
three aspects of elastic matrix assembly are therefore key areas areas of research interest in the field today. Further progress in enabling biomimetic elastic matrix assembly requires advances in scaffold development, which are critical since scaffolds provide the substrata for cell adherence, spreading, and proliferation, include the biomechanical transductive cues that regulate cell behavior, and serve as templates for cellular deposition of extracellular matrix (ECM).

\section{Improving elastin precursor recruitment and crosslinking}

As we have stated in the introduction, there are several growth factors and other biomolecular agents that are now known to stimulate tropoelastin synthesis by adult cells. However, efficient recruitment and crosslinking of these precursors into an elastic matrix can be more difficult to achieve, and in fact represent a more critical problem, which if addressed can have significantly greater impact than improving tropoelastin synthesis itself. For example, Mecham et al. [13] demonstrated in vitro that fetal bovine ligamentum nuchae fibroblasts produce tropelastin of which most was released into the culture media instead of being incorporated into a matrix. Our studies have further shown that while healthy adult rat aortic smooth muscle cells typically incorporate only $8-15 \% \mathrm{w} / \mathrm{w}$ of synthesized tropoelastin precursors into a matrix, [18] this fraction can be even lower at $1-2 \%$ $\mathrm{w} / \mathrm{w}$ for cells isolated from diseased (e.g., induced abdominal aortic aneurysms) rat [25], and human aortae [26]. This implies that $85-98 \%$ $\mathrm{w} / \mathrm{w}$ of synthesized tropoelastin is untapped in generating elastic matrix structures. The corollary is that a tremendous potential for harnessing otherwise unused tropoelastin precursor molecules for creating matrix structures exist, provided appropriate strategies are identified to enhance their recruitment, crosslinking, and further organization into structures that can influence tissue elasticity.

Substrate hydrophilicity and anionicity appear to significantly benefit elastin precursor recruitment. For example, it has been shown that polyhydroxy alkaonate (PHA) scaffolds incorporating the hydrophilic 4-hydroxybutyrate (4-HB) moiety stimulate increased cell adherence and insoluble elastin deposition relative to similar scaffolds incorporating the more hydrophobic hydroxyhexaonate (HHx) instead [27]. The increases were also determined to correlate positively to increases in 4-HB content, and thereby, hydrophilicity. Similarly, preferred tropoelastin coacervation and crosslinking has been noted in cultures containing exogenous supplements of anionic long-chain hyaluronan (HA), [28] a matrix glycosaminoglycan (GAG), and hydrogels and other biomaterials incorporating derivatized and/ or crosslinked long-chain HA. [29-31] Gheduzzi et al. [32] provided some insight into the mechanisms underlying these phenomena in demonstrating that negatively charged proteoglycans (e.g., heparin sulfate or HS) serve to stabilize globular coacervates of tropoelastin precursors that reduces the minimal concentration of tropoelastin required to initiate elastic microfiber assembly. Less anionic proteoglycans however (e.g. chondroitin sulfate or CS) do not have the same effect, either due to charge effects or specific molecular interactions [33]. The coacervates are then released in a timed manner when the positive charge on tropoelastin is neutralized upon crosslinking by lysyl oxidase (LOX) [34]. This allows the coacervates to move through the ECM and engage glycoprotein microfibrils for fiber assembly. Thus, a potential strategy to improve elastin precursor recruitment for matrix assembly might be to select anionic scaffolding materials, or modify scaffold surfaces with negatively charged functional groups to enhance electrostatic interactions with the positively charged elastin precursors. An alternative strategy might be to incorporate negatively charged biomolecules (e.g., HS) themselves into the scaffolds, for the same purpose.

There is also strong evidence that the composition of the ECM microenvironment within scaffolds influences the extent and timing of elastin precursor release from the cell surface, their recruitment and crosslinking to form stable coacervates, and the engagement of microfibrillar proteins to form elastic fibers. For example, compared to neonatal vascular SMCs, adult SMCs produce significantly higher amounts of proteoglycans such as versican. Hinek et al. [6] have shown that the CS side chains on the versican molecule overwhelmingly engage the galactolectin domain of the elastin binding protein on the cell surface, reducing engagement of the same site by microfibrillar glycoproteins such as fibrillin that form pre-scaffolds for elastin deposition. This leads to diminished tropoelastin affinity for the EBP and its premature release. The result is poor coacervation and crosslinking of tropoelastin and its diminished deposition onto the glycoprotein pre-scaffolds. HS however does not elicit these negative effects since it does not contain galactose sugars and cannot bind the EBP. The presence of long-chain HA at the cell surface also enables versican binding via its HA binding motif, followed by engagement of the EBP by its CS chains as stated above. Reducing versican bioavailability at the surface via incorporation of splice variants lacking the CS-bearing domains, as shown by Merrilees et al. [35] or potentially preventing versican binding to long-chain HA by reducing availability of long-chain HA at the cell surface through competitive engagement of the HA receptor (i.e. CD44) by much shorter, non-versican-binding HA oligomers (HA-o), [36] may be two possible strategies to enhance fractional elastic matrix yield in culture.

In the absence of proper crosslinking, elastin incorporated into tissue-engineered constructs resembles disorganized amyloid elastic fibers, which are typically produced in vivo in certain pathological conditions (e.g. atherosclerosis). Various strategies have shown potential to improve crosslinking, including providing media supplements of lysyl-oxidase (LOX) and LOX activity-enhancers (e.g. copper ions in the form of soluble copper salts). Recently, Kothapalli and Ramamurthi [37] showed that controlled and gradual copper ion release from copper nanoparticles prevents long-term cytotoxicity associated with delivery of high doses of soluble copper salts, while significantly increasing the fraction of synthesized elastin precursors that are incorporated into the matrix. With these promising outcomes, research in the field is now increasingly directed at addressing another key challenge, which is being able to align elastic fibers into fiber bundles and orient these bundles in preferred directions characteristic of targeted tissue types. This is critical to create tissues demonstrating anisotropic mechanics which uniquely influence behavior of contacting parenchymal cells within the constructs via biomechanical transduction.

\section{The role of scaffolds to promote alignment and direct orientation of elastic fibers}

Alignment of elastic fibers and their spatial orientation may be guided through culture of cells on scaffolds with appropriate surface topography and 3-dimensional (3-D) architecture. Scaffolds present cues and substrates for cell adherence, spreading, and proliferation, serve as a 3-D template for matrix deposition by seeded cells, and function as the primary load-bearing components of growing/maturing tissue constructs till this role is assumed by the generated matrix structures. Through the choice of a type of macromolecule (i.e. natural 
or synthetic) and a processing technique, the resulting biomaterial can be customized to present the desired surface chemistry, topography, mechanics, and other cues that have been shown to influence cellular generation of tropoelastin and cell-mediated assembly and maturation of elastic fibers.

Topographic features present on the scaffolds provide one of the most important clues to promote matrix alignment. A significant body of work indicates that most anchorage-dependant cells (e.g., SMCs, fibroblasts, and mesenchymal stem cells) attach, spread, and align along aligned surface features (e.g. microgrooves and electrospun fibers) of cellular dimensions, via contact guidance. Studies, such as that by Lee et al. [38] have shown that cells (e.g., ligament fibroblasts) oriented in such a manner also deposit matrix in the same direction, which is not achieved with cell culture on smooth tissue culture plastic [39]. Most literature in this area pertains to organization of aligned collagen fibers by cells. Although the exact role of the cell in alignment of collagen is not completely clear, the presence of cells does not appear to be required for self-assembly of collagen precursors after cleavage of the end pro-peptides [40]. Likewise, thermo-responsive self-assembly of elastin-like peptides has been demonstrated [41]. However, production of aligned collagen fibrils does require an additional input such as direct or indirect cell involvement. Canty et al. [42] have shown that plasma-membrane structures called fibripositors are co-aligned with collagen fibrils, and they proposed that these structures direct collagen fibril alignment. It is quite possible that cellular mechanisms also exist for alignment of elastic fibrils, although the role of the cells and contact guidance for these fibers is even less elucidated.

In the case of elastic fibers, cells appear to play a role in enabling their alignment. Rossi et al. have shown that endothelial cells elongate along surface grooves and deposit fibrillin microfibrils that are aligned along the major axis of polarization of the cells [43]. Fibrillin is a glycoprotein that assembles to form macromolecules, which in selfassembly experiments are typically not in the extended, oriented state that they are in intact tissue [44]. Baldock et al. have proposed that cells play a role in alignment, with the monomers associating with the amine and carboxyl groups present on the cell surface and then assembling to form microfibrils [45]. Interaction with the cell membrane may also play a role in the initial steps of tropoelastin aggregation, as Kozel et al. have shown.[46] However, the elastin globules that deposit on the glycoprotein microfibril scaffolds to ultimately form mature elastic fibers, do not exhibit preferred orientation, or alignment between themselves as do the microfibrils. In light of the benefits of copper ion releasing nanoparticles to enhancing elastin precursor crosslinking, as we discussed earlier, patterning these nanoparticles on scaffold surfaces could be a useful strategy to guide orientation and growth of newly-crosslinked elastin deposits and promote alignment of elastic fibrils. Overall, the presence of aligned topographic features on scaffold surfaces appears to be a reliable strategy to spatially orient and align the microfibrils, and thereby the elastic fibers that are assembled.

Another strategy to direct matrix cell/matrix alignment is through patterning a surface with cell adhesion peptides. This technique has been used extensively to direct cell and collagen orientation, but its application to elastic fibers has been limited. However, Patel et al. [47] demonstrated in a recent publication that engineered synthetic peptides, containing cell adhesion and cross-linking domains found in natural elastin, induce ECM deposition and crosslinking. The cells generated tropoelastin precursors when they were cultured on the synthetic-peptide immobilized scaffold surfaces. This strategy to enhance elastic matrix deposition on synthetic scaffolds can potentially be used to direct elastic fiber alignment and spatial orientation as well, by bioprinting a template of nano-patterned synthetic elastin peptides on the scaffold surface. However, the major limitation of this technique may be scaling it up to $3-\mathrm{D}$, clinically implantable grafts.

\section{Conclusion}

There are clearly multiple challenges to engineering an elastic fiber-rich tissue constructs. With the long-standing challenge of increasing troproelastin synthesis by elastogenically deficient adult cell types having been addressed via multiple approaches, with some success, the focus now lies in tackling more critical issues which are poor recruitment and crosslinking of elastin precursors and the need to organize the matrix deposits into mechanically-compliant structures. While the biomaterial scaffold forms only one component of the cellular microenvironment, it provides one of the most potent cues to induce mature matrix production as well directing orientation of elastic matrix structures, another important challenge. Elastogenic benefits of hydrophilic and anionic surfaces as well as surfaces that prevent CS binding have been described in this editorial. In addition, while the scaffold/ cellular role in matrix alignment is still being elucidated, it is appears that aligned topographic features are currently the best method to direct alignment of cell-generated elastic matrix. While progress has been made, adopting new strategies such as aligned topographic cues, scaffold chemistries and specific cell-binding sequences, and surfacepresented/patterned biomolecules, or combinations of these strategies together with the use of other cell types (e.g. mesenchymal, embryonic, or induced pluripotent stem cells) that are potentially more elastogenic than are parenchymal cells from mature, adult tissues, will likely determine future progress towards overcoming the current challenges to biomimetic elastic matrix engineering.

\section{References}

1. Li DY, Brooke B, Davis EC, Mecham RP, Sorensen LK, et al. (1998) Elastin is an essential determinant of arterial morphogenesis. Nature 393: 276-280.

2. Li DY, Faury G, Taylor DG, Davis EC, Boyle WA, et al. (1998) Novel arteria pathology in mice and humans hemizygous for elastin. J Clin Invest 102: 17831787.

3. Faury G, Garnier S, Weiss AS, Wallach J, Fulop T Jr, et al. (1998) Action of tropoelastin and synthetic elastin sequences on vascular tone and on free $\mathrm{Ca} 2+$ level in human vascular endothelial cells. Circ Res 82: 328-336.

4. Robert L, Jacob MP, Fulop T (1995) Elastin in blood vessels. Ciba Found Symp 192: 286-303.

5. Kielty CM, Sherratt MJ, Shuttleworth CA (2002) Elastic fibres. J Cell Sci 115 2817-2828.

6. Hinek A, Mecham RP, Keeley F, Rabinovitch M (1991) Impaired elastin fiber assembly related to reduced $67-\mathrm{kD}$ elastin-binding protein in fetal lamb ductus arteriosus and in cultured aortic smooth muscle cells treated with chondroitin sulfate. J Clin Invest 88: 2083-2094.

7. Johnson DJ, Robson P, Hew Y, Keeley FW (1995) Decreased elastin synthesis in normal development and in long-term aortic organ and cell cultures is related to rapid and selective destabilization of mRNA for elastin. Circ Res 77: 11071113.

8. McMahon MP, Faris B, Wolfe BL, Brown KE, Pratt CA, et al. (1985) Aging effects on the elastin composition in the extracellular matrix of cultured rat aortic smooth muscle cells. In Vitro Cell Dev Biol 21: 674-680.

9. Wognum S, Schmidt DE, Sacks MS (2009) On the mechanical role of de novo synthesized elastin in the urinary bladder wall. J Biomech Eng 131:101018.

10. Rahn DD, Acevedo JF, Word RA (2008) Effect of vaginal distention on elastic fiber synthesis and matrix degradation in the vaginal wall: potential role in 
the pathogenesis of pelvic organ prolapse. Am J Physiol Regul Integr Comp Physiol 295: R1351-1358.

11. Fornieri C, Quaglino D Jr, Mori G (1992) Role of the extracellular matrix in age-related modifications of the rat aorta. Ultrastructural, morphometric, and enzymatic evaluations. Arterioscler Thromb 12: 1008-1016.

12. Mecham RP, Levy BD, Morris SL, Madaras JG, Wrenn DS (1985) Increased cyclic GMP levels lead to a stimulation of elastin production in ligament fibroblasts that is reversed by cyclic AMP. J Biol Chem 260: 3255-3258.

13. Mecham RP, Lange G, Madaras J, Starcher B (1981) Elastin synthesis by ligamentum nuchae fibroblasts: effects of culture conditions and extracellular matrix on elastin production. J Cell Biol 90: 332-338.

14. Wolfe BL, Rich CB, Goud HD, Terpstra AJ, Bashir M, et al. (1993) Insulin-like growth factor-I regulates transcription of the elastin gene. J Biol Chem 268: 12418-12426.

15. Sales VL, Engelmayr GC Jr, Mettler BA, Johnson JA Jr, Sacks MS, et al. (2006) Transforming growth factor-beta1 modulates extracellular matrix production, proliferation, and apoptosis of endothelial progenitor cells in tissue-engineering scaffolds. Circulation;114: 193-199.

16. Mitts TF, Bunda S, Wang Y, Hinek A (2010) Aldosterone and mineralocorticoid receptor antagonists modulate elastin and collagen deposition in human skin. J Invest Dermatol 130: 2396-2406.

17. Tsukahara K, Takema Y, Fujimura T, Moriwaki S, Kitahara T, et al. (1999) Alltrans retinoic acid promotes the repair of tortuosity of elastic fibres in rat skin. Br J Dermatol 140: 1048-1053.

18. Joddar B, Ramamurthi A (2006)Elastogenic effects of exogenous hyaluronan oligosaccharides on vascular smooth muscle cells. Biomaterials 27: 56985707.

19. Long JL, Tranquillo RT (2003) Elastic fiber production in cardiovascular tissueequivalents. Matrix Biol 22: 339-350.

20. Li S, Lao J, Chen BP, Li YS, Zhao Y, et al. (2003) Genomic analysis of smooth muscle cells in 3-dimensional collagen matrix. FASEB J 17: 97-99.

21. Kim BS, Mooney DJ (1998) Engineering smooth muscle tissue with a predefined structure. J Biomed Mater Res 41: 322-332.

22. Crapo PM, Wang Y (2010 ) Physiologic compliance in engineered smalldiameter arterial constructs based on an elastomeric substrate. Biomaterials 31: $1626-1635$

23. Kim BS, Nikolovski J, Bonadio J, Mooney DJ (1999) Cyclic mechanical strain regulates the development of engineered smooth muscle tissue. Nat Biotechnol 17: $979-983$.

24. Bashur CA, Ramamurthi A Aligned Electrospun Scaffolds and Elastogenic Factors for Vascular Cell-mediated Elastic Matrix Assembly. J Tissue Eng Regen Med in press.

25. Gacchina CE, Deb P, Barth JL, Ramamurthi A (2011) Elastogenic inductability of smooth muscle cells from a rat model of late stage abdominal aortic aneurysms. Tissue Eng Part A 17: 1699-1711.

26. Gacchina C, Brothers T, Ramamurthi A (2011) Evaluating smooth muscle cells from $\mathrm{CaCl}$-induced rat aortal expansions as a surrogate culture model for study of elastogenic induction of human aneurysmal cells. Tissue Eng Part A 17: $1945-1958$

27. Cheng ST, Chen ZF, Chen GQ (2008) The expression of cross-linked elastin by rabbit blood vessel smooth muscle cells cultured in polyhydroxyalkanoate scaffolds. Biomaterials 29: 4187-4194.

28. Joddar B, Ramamurthi A (2006) Fragment size- and dose-specific effects of hyaluronan on matrix synthesis by vascular smooth muscle cells. Biomaterials 27: 2994-3004.

29. Ramamurthi A, Vesely I (2003) Ultraviolet light-induced modification of crosslinked hyaluronan gels. J Biomed Mater Res A 66: 317-329.

30. Ibrahim S, Joddar B, Craps M, Ramamurthi A (2007) A surface-tethered model to assess size-specific effects of hyaluronan (HA) on endothelial cells. Biomaterials 28: 825-835.

31. Ibrahim S, Kothapalli CR, Kang QK, Ramamurthi A (2011) Characterization of glycidyl methacrylate - crosslinked hyaluronan hydrogel scaffolds incorporating elastogenic hyaluronan oligomers. Acta Biomater 7: 653-665.
32. Gheduzzi D, Guerra D, Bochicchio B, Pepe A, Tamburro AM, et al. (2005) Heparan sulphate interacts with tropoelastin, with some tropoelastin peptides and is present in human dermis elastic fibers. Matrix Biol 24: 15-25.

33. Broekelmann TJ, Kozel BA, Ishibashi H, Werneck CC, Keeley FW, et al. (2005) Tropoelastin interacts with cell-surface glycosaminoglycans via its $\mathrm{COOH}$ terminal domain. J Biol Chem 280: 40939-40947.

34. Fornieri C, Baccarani-Contri M, Quaglino D Jr, Pasquali-Ronchetti I (1987) Lysyl oxidase activity and elastin/glycosaminoglycan interactions in growing chick and rat aortas. J Cell Biol 105: 1463-1469.

35. Merrilees MJ, Lemire JM, Fischer JW, Kinsella MG, Braun KR, et al. Retrovirally mediated overexpression of versican $\mathrm{v} 3$ by arterial smooth muscle cells induces tropoelastin synthesis and elastic fiber formation in vitro and in neointima after vascular injury. Circ Res 90: 481-487.

36. Joddar B, Ibrahim S, Ramamurthi A (2007) Impact of delivery mode of hyaluronan oligomers on elastogenic responses of adult vascular smooth muscle cells. Biomaterials 28: 3918-3927.

37. Kothapalli CR, Ramamurthi A (2009) Copper nanoparticle cues for biomimetic cellular assembly of crosslinked elastin fibers. Acta Biomater 5: 541-553.

38. Lee CH, Shin HJ, Cho IH, Kang YM, Kim IA, et al. (2005) Nanofiber alignment and direction of mechanical strain affect the ECM production of human ACL fibroblast. Biomaterials 26: 1261-1270.

39. L'Heureux N, Paquet S, Labbe R, Germain L, Auger FA (1988) A completely biological tissue-engineered human blood vessel. FASEB J 12: 47-56.

40. Kadler KE, Holmes DF, Trotter JA, Chapman JA. Collagen fibril formation Biochem J 316: 1-11.

41. Pechar M, Brus J, Kostka L, Konak C, Urbanova M, et al. (2007) Thermoresponsive self-assembly of short elastin-like polypentapeptides and their poly(ethylene glycol) derivatives. Macromol Biosci 7: 56-69.

42. Canty EG, Starborg T, Lu Y, Humphries SM, Holmes DF, Meadows RS, Huffman A, O'Toole ET, Kadler KE. Actin filaments are required for fibripositormediated collagen fibril alignment in tendon. J Biol Chem 281: 38592-38598.

43. Rossi A, Pasqui D, Barbucci R, Gerli R, Weber E (2009) The topography of microstructured surfaces differently affects fibrillin deposition by blood and lymphatic endothelial cells in culture. Tissue Eng Part A 15: 525-533.

44. Kielty CM, Baldock C, Lee D, Rock MJ, Ashworth JL, et al. ( 2002) Shuttleworth CA. Fibrillin: from microfibril assembly to biomechanical function. Philos Trans R Soc Lond B Biol Sci 357: 207-217.

45. Baldock C, Koster AJ, Ziese U, Rock MJ, Sherratt MJ, et al. (2001) The supramolecular organization of fibrillin-rich microfibrils. J Cell Biol 152: 10451056

46. Kozel BA, Rongish BJ, Czirok A, Zach J, Little CD, et al. (2006) Elastic fiber formation: a dynamic view of extracellular matrix assembly using timer reporters. J Cell Physiol 207: 87-96

47. Patel D, Menon R, Taite LJ (2011) Self-assembly of elastin-based peptides into the ECM: the importance of integrins and the elastin binding protein in elastic fiber assembly. Biomacromolecules 12: 432-440. 